\title{
The Emergency Implant: Immediate Extraction Replacement in the Esthetic Zone
}

\author{
Jack Hahn
}

Private Practice, 910 Barry Lane, Cincinnati, Ohio-452 29

Correspondence: Jack Hahn, Private Practice, 910 Barry Lane, Cincinnati, Ohio-45229, e-mail: replace7@mac.com

\begin{abstract}
As more and more patients present to dental offices requiring extraction and replacement of teeth in the esthetic zone, the pro ffered treatment options range from a removable prosthesis as a temporary measure to three unit bridges and implants. Of these modalit ies, more and more patients prefer the implant option especially if it can be performed immediately. The author has placed and restøed 2700 immediate implants in the 13 years per iod between 1996 and 2009 and discusses the principles of immediate implants.
\end{abstract}

Keywords: Immediate implant, fresh extraction sockets.

More patients are presenting themselves to dental offices in hopes for an immediate replacement for a tooth in the esthetic zone that is no longer restorableor fractured. Many of these teeth have had endodontic treatment and are fractured. When presenting the patient of their options such as a temporary partial (flipper), three unite fixed bridge or an implant. Most choose the implant, especially if it can be done immediately. Since, March 1996 to September of 2009, this author has placed and restored over 2700 of immediate implants in the esthetic zone, premolar to premolar, with less than 5\% exhibiting complication or failure. Like all modalities in dentistry, we must adhere to certain principals when diagnosing and performing immediate replacement procedures. This clinician, as well as many others, have discovered these principals from their individual clinical experiences. Beginning August of 1989 to August 2004, this author participated in a multicenter study of immediate extraction implant replacement with an immediately placed provisional restoration. A total of four clinicians participated in the study, tracking the majority of the implants a minimum of 12 years. The compiled was $96.7 \%$ success using the accepted published standards for success.

The basic principals to achieving predictable success in immediate replacement implant dentistry are:

1. Nontraumatic extraction.

2. Remove any residual pathology in the socket (may sterilize with a laser).

3. Place $2 \mathrm{~mm}$ twist drill at the apex of the socket and slide it at least $2 \mathrm{~mm}$ to the palatal aspect.
4. Drag a periodontal currett along the labial or fascial wall to discover any fenestrations. Fenestration of 5 $\mathrm{mm}$ in diameter or more, it is recommendd to perform a flap procedure. Less than $5 \mathrm{~mm}$. It is preferred not to flap to keep the periosteium on the bone to help maintain blood supply. If there is an absence of the labial plate, in such cases it is advised to graft and no Implant Placement. Implant place 5 months postextraction-delayed extraction replacement. Also, delayed placement is recommended if there is active infection, purulent exudate. Patient is place on a 5 days course of antibiotic and implant placed 3 to 4 weeks postextraction.

5. Choose an implant diameter and prepare the socket so that there is a space of at least $1 \mathrm{~mm}$ between theabial or fascial bone. Starting the socket prepar ation $2 \mathrm{~mm}$ to the palatal aspect will help to accomplish avoiding contact or pressure against the extremely thin labial bone. Spaces or gaps or $2 \mathrm{~mm}$ or more should be grafted with a soft bone putty, example Nova bone or Pepgen flow.

6. If there is sufficient bone between existing socket apex and nasal or maxillary sinus, select an implant that is 1 to $2 \mathrm{~mm}$ longer than the too that is being replaced.

7. Use screw or threadformer against the de nser palatal wall of the socket in order to prevent the implant from being influenced and pushed against the thin labial bone plate. 
Use a torque to determine primary stability. This author primarily places replace select (Nobel Biocare). There for thi s system to determine primar y stability for immediate funciton is between 35 to $45 \mathrm{nu} / \mathrm{cm}$.

8. Abutment choices can be of the clinician's preference. In most cases this clinician prefers temporary abutments, which can be removed after the integration period, implant level transfer impression recorded and the laboratory fabricates the fina 1 abutment and restoration. If final abutments are placed at the time of insertion, ceramic abutments are to be prepared using a high speed drill, diamonds with irrigation. Titanium may be prepared using high speed, sharp metal cutting burs with copious amounts of water.

9. The provisional restoration must be well adapted and adjusted at least $1 \mathrm{~mm}$ out of oc clusion in all excursions. A retentive cement is recommended to insure that there is no movement of the provisional restoration. Ex. Improv. Care must be taken to remove any excess cement. If soft tissue manipulations were performed, it is generally recommended to suture after cementation. Patient are instructed to avoid functioning on the site, prescribed a 5 days course of antibiotics and analgesics.

It is this authors experience that the patients are extremely satisfied with immediate implant treatment and recommend other new patients to the practice. In today's world economic environment, immediate extraction implant replacement is a highly accepted option. The basic principals that have been described must be adhered to in order to insure success. This clinician prefers tapered implants for extraction replacements as they are more anatomically suitable to alveolar socket forms and studies show that they are initially more stable at the time of placement which is important for immediate function.

Placement of dental implants into fresh extraction sites offers a number of significant advantages to both the patients and the clinicians. Without the support provided by the functioning dental units ( e.g., teeth, implants), the bony receptor sites soon undergo a catabolic phases that results in varying amounts of atrophy after tooth removal. Recession of the mucosal and gingival tissues accompanies and follows changes in the alveolar ridge. As a result, achievement of superior esthetics be comes more di fficult, particularly in the esthetic zone of the anterior maxilla.

Further, implants in fresh extraction sites can be placed in the same position as the extracted tooth. Immediate implant placement facilitates the final restoration and minimizes the need for severely angled abutments or fabrication of telescopic copings. When necessary, the surgeon can position the implant more favorably than the original tooth by redirecting the burs when preparing the implant receptor site. With the extraction socket as a guide, the surgeon can also more easily determine the appropriate parallelism and alignment relative to the opposing and adjacent residual dentition and to adjacent $\mathrm{i}$ mplants when there are multiple extractions and implants. Improved final function and esthetics typically result.

Finally, elimination of the once-standard ${ }^{1-7}$ wait for primary healing of the soft tissues and the regeneration of the osseous structure significantly shorten the overall treatment time and $t$ he interval $d$ uring which the patient must live in a transitional state with or without teeth. Consequently, more patients accept treatment. The consolidation of procedures has also proven to increase the overall costeffectiveness of cases. Although some of the early implant practitioners assumed that the long-term implant survival depended on placement in healed alveolar ridge(s), a growing body of evidence confirms that immediate postextraction implant placement yields excellent and predictable results.

\section{GUIDELINES FOR EXTRACTIONWHEN PLANNING FOR IMMEDIATE IMPLANT PLACEMENT}

The following guidelines for extractions are provided when planning for immediate placement of implants.

1. Preoperative evaluation.

2. Antibiotic therapy initiation.

3. Preservation of the bony receptor site.

4. Procedural delays.

5. Avoidance of excessive pressure.

6. Osteotomy preparation.

7. Improvements for placements.

8. Bone grafts.

9. Soft tissue closure.

10. Successful osseointegration.

11. Implant loading.

\section{Preoperative Evaluation}

Patients should be thoroughly evaluated before all elective procedures. However, acute situations such as those involving a nonrestorable fractured tooth or those with pulpal exposure, may not allow for preliminary evaluation. 


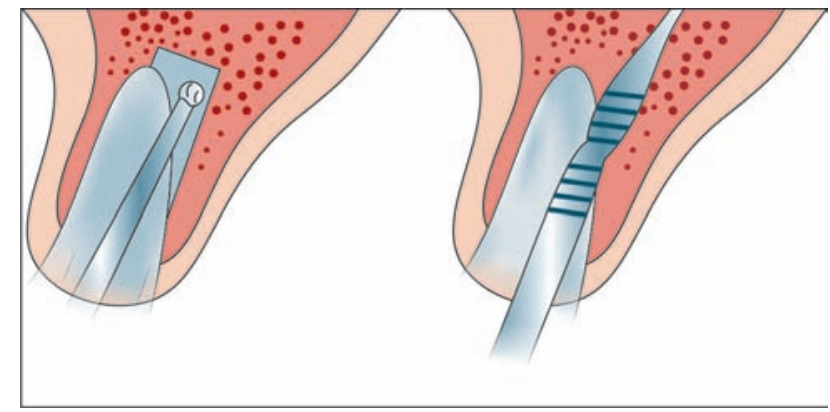

Fig. 1: $2 \mathrm{~mm}$ twist drill

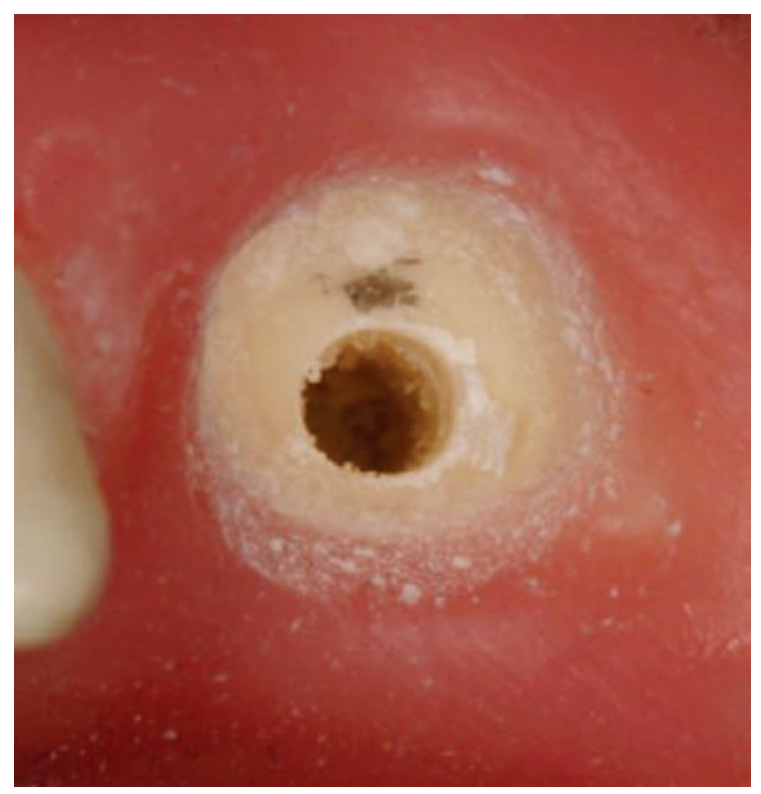

Fig. 2: Preparation to the palatal aspect

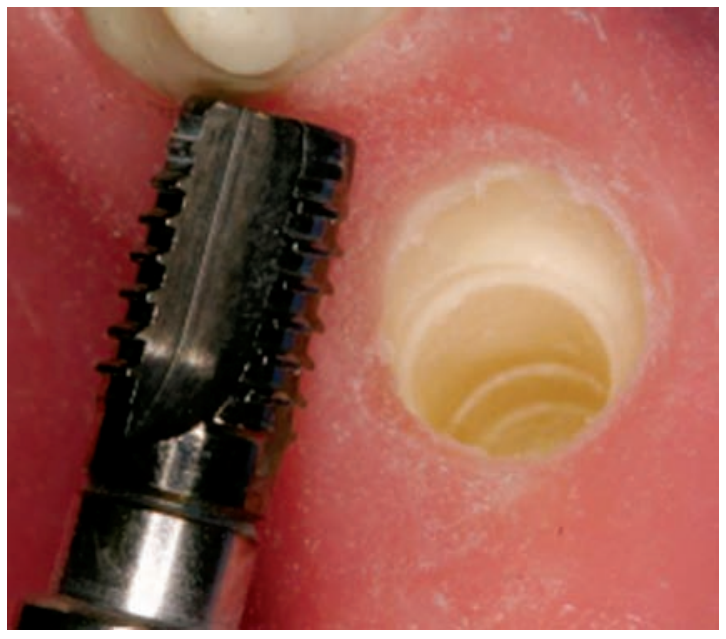

Fig. 3: Threadformer

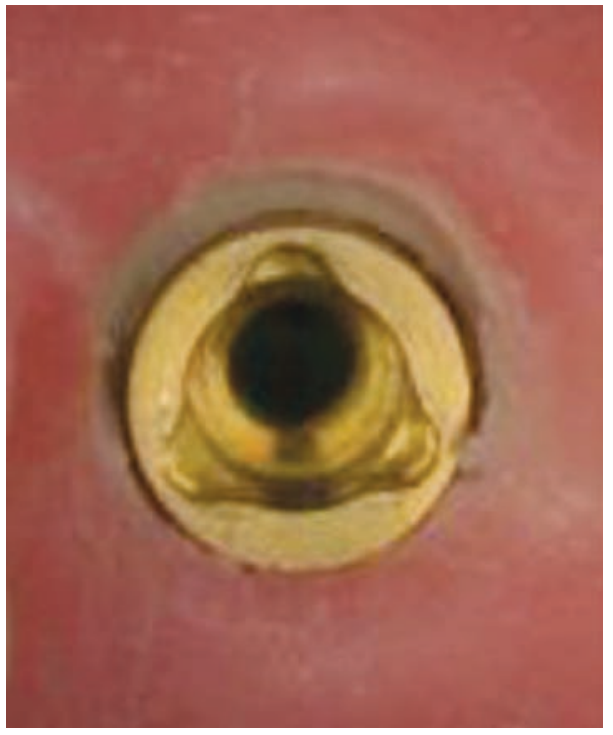

Fig. 4: Implant spacing

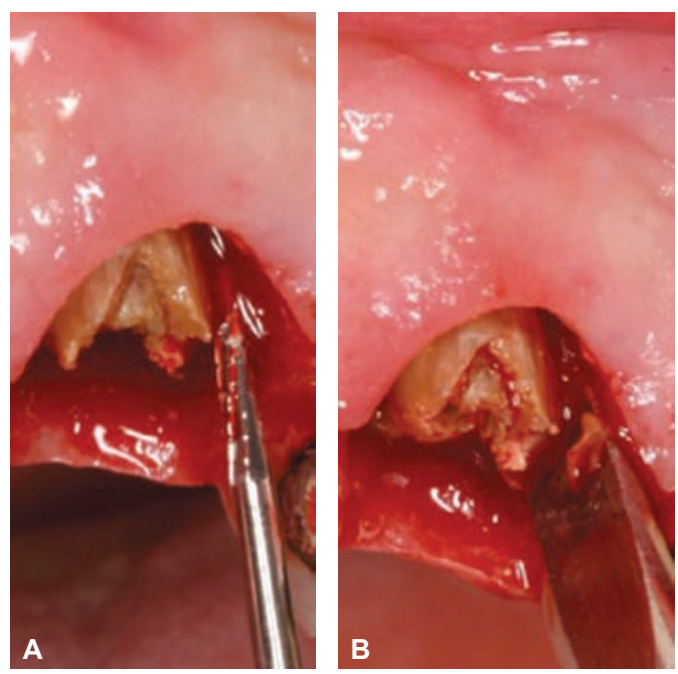

Figs 5A and B: (A) $700 \mathrm{xxl}$ bur (B) Elevator
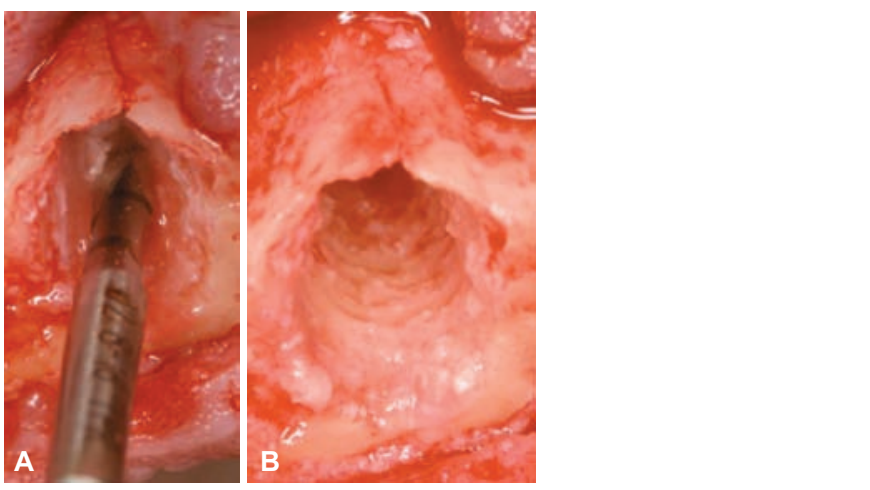

Figs 6A to C: (A) $2 \mathrm{~mm}$ twist drill (B) Palatal wall (C) Impl ant 


\section{CASE 1}
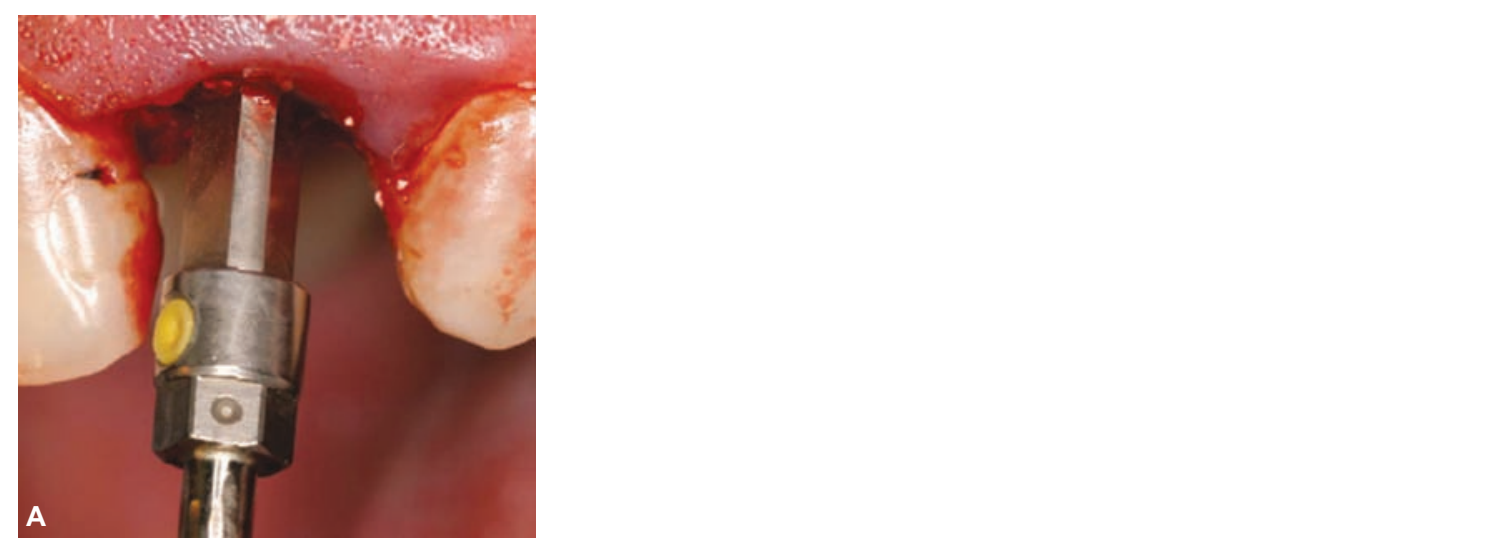

Figs $7 \mathrm{~A}$ and $\mathrm{B}: 45 \mathrm{nu} / \mathrm{cm}$
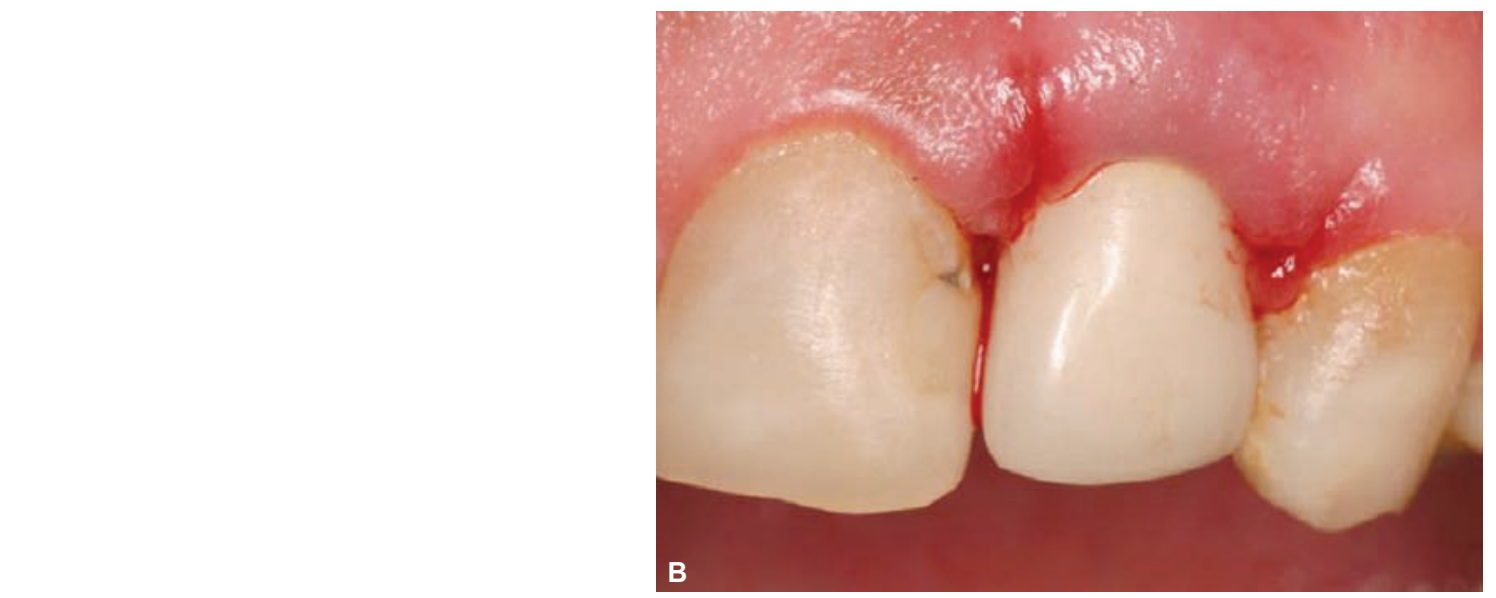

Figs 8A and B: Ten days postoperative
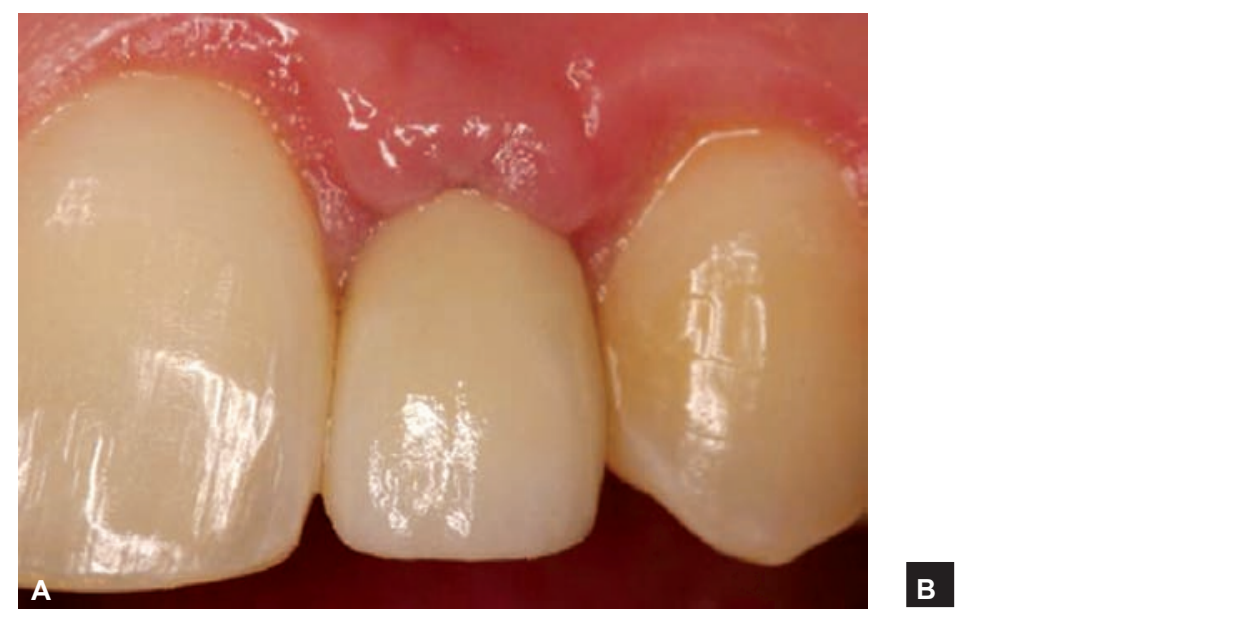

Figs 9A and B: One year 


\section{CASE 2}

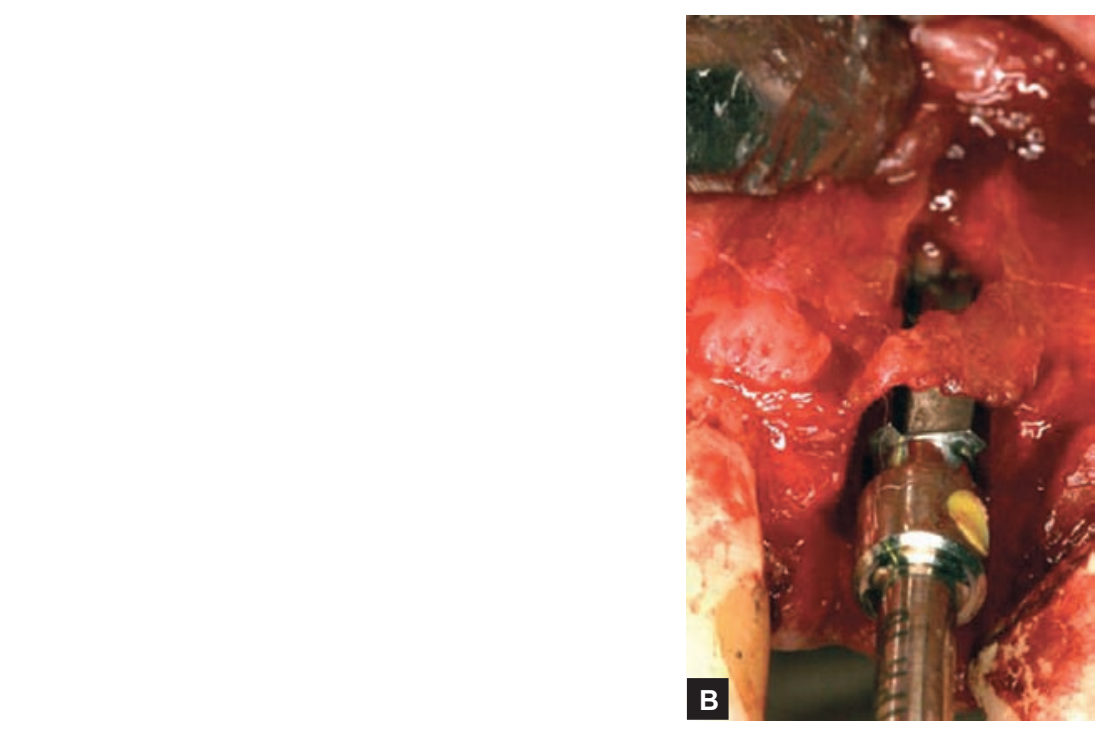

Figs $10 A$ and $B: 4.3$ drill, note space on fascial

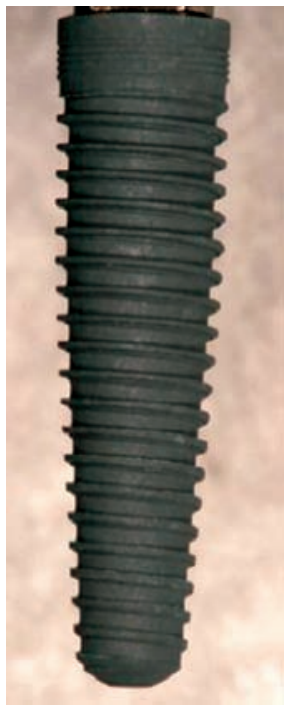

Fig. 11: Replace select tapered groovy

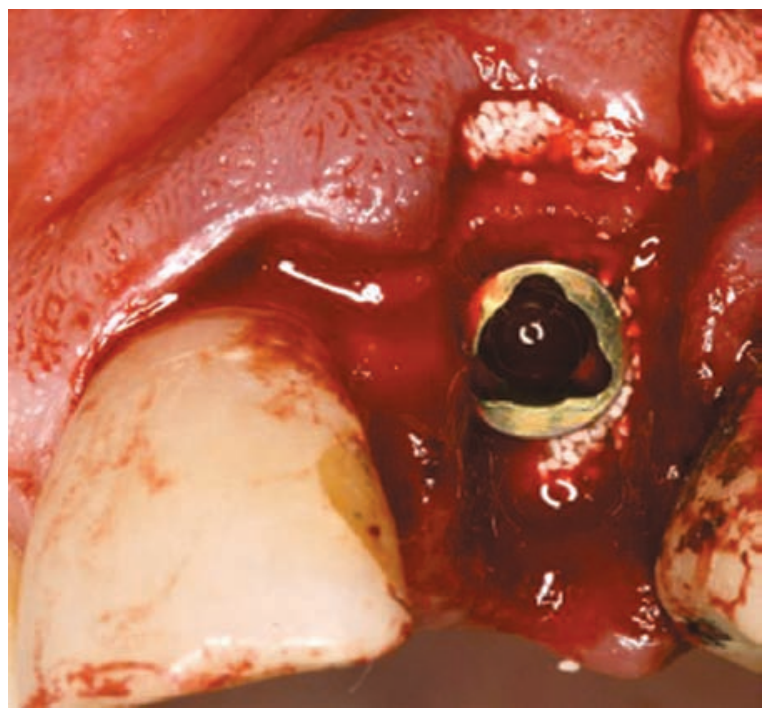

Fig. 12: Implant placement

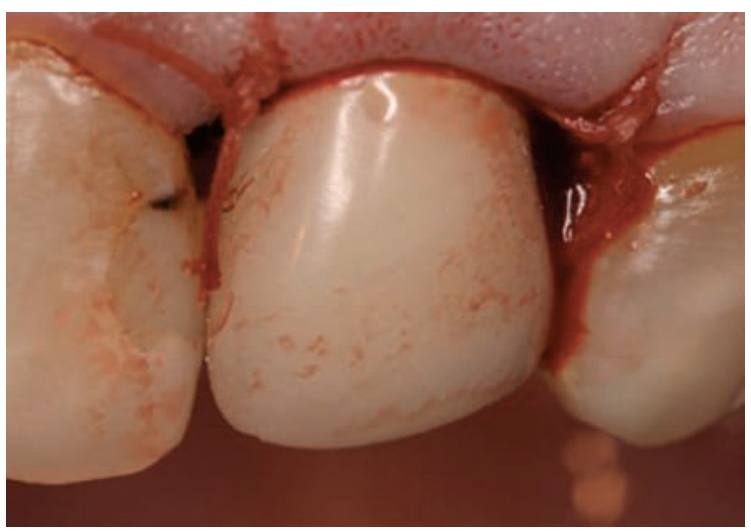

Fig. 13: Provisional restoration 


\section{CASE 3}
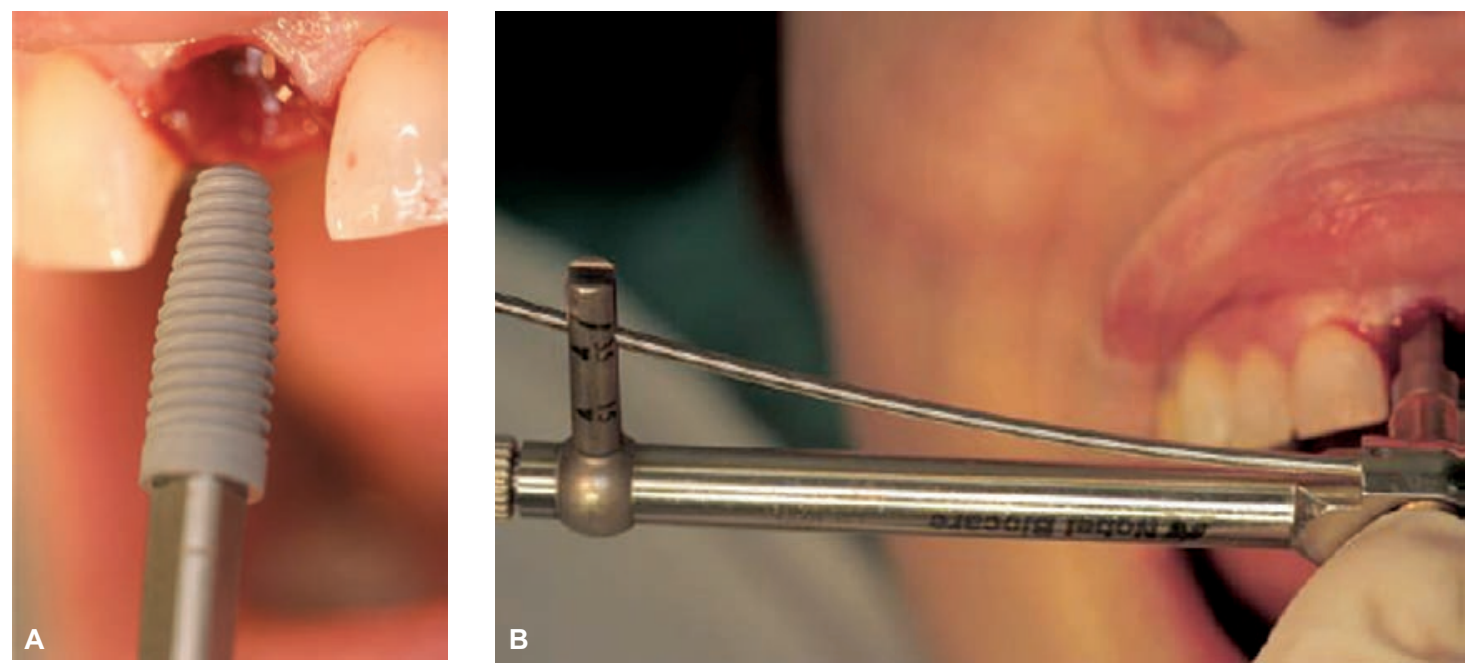

Figs 14A and B: (A) Replace tapered groovy (B) Insertion wrench

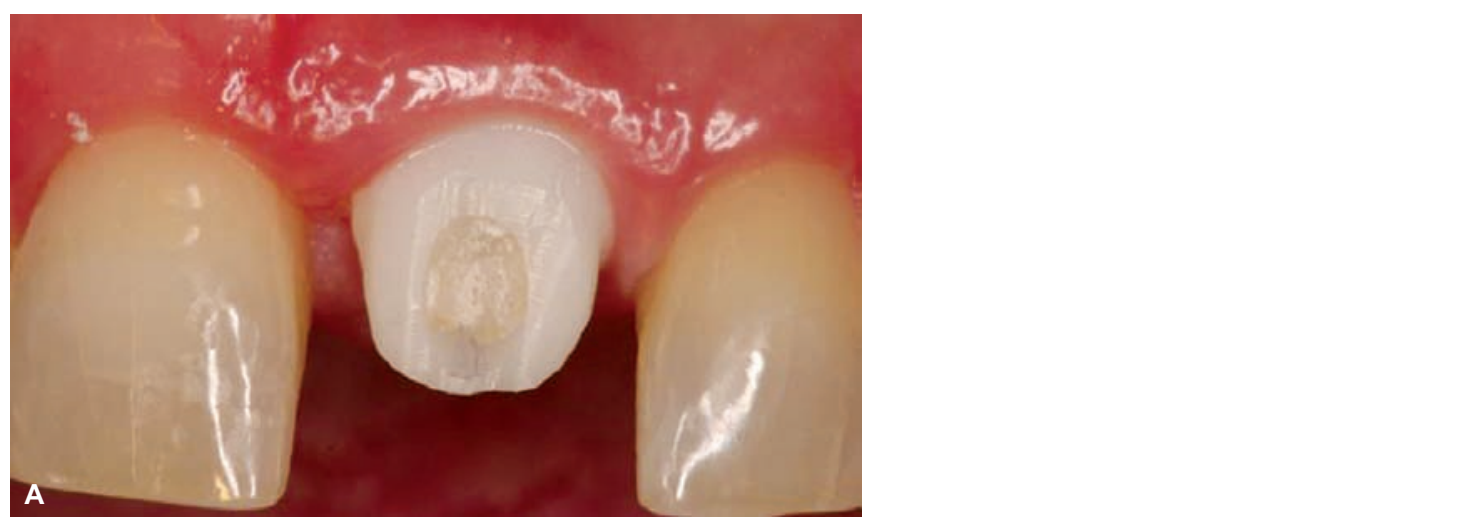

Figs 15A and B: Ceramic abutment and temporary

\section{CASE 4}
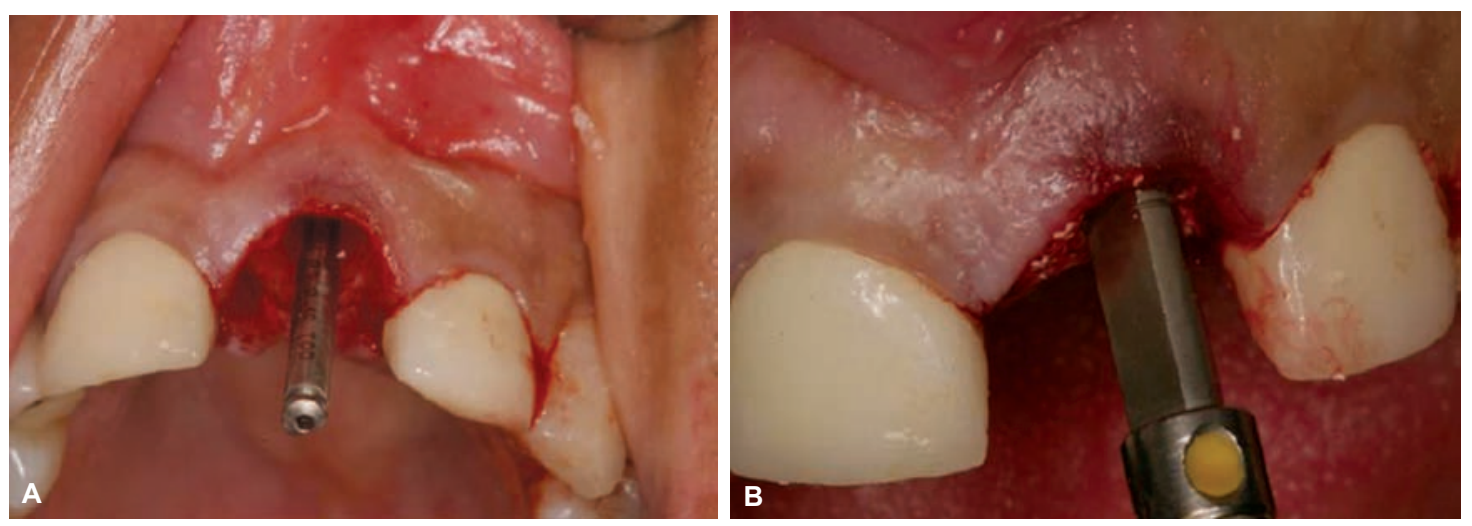

Figs 16A and B: Osteotomy preparation 

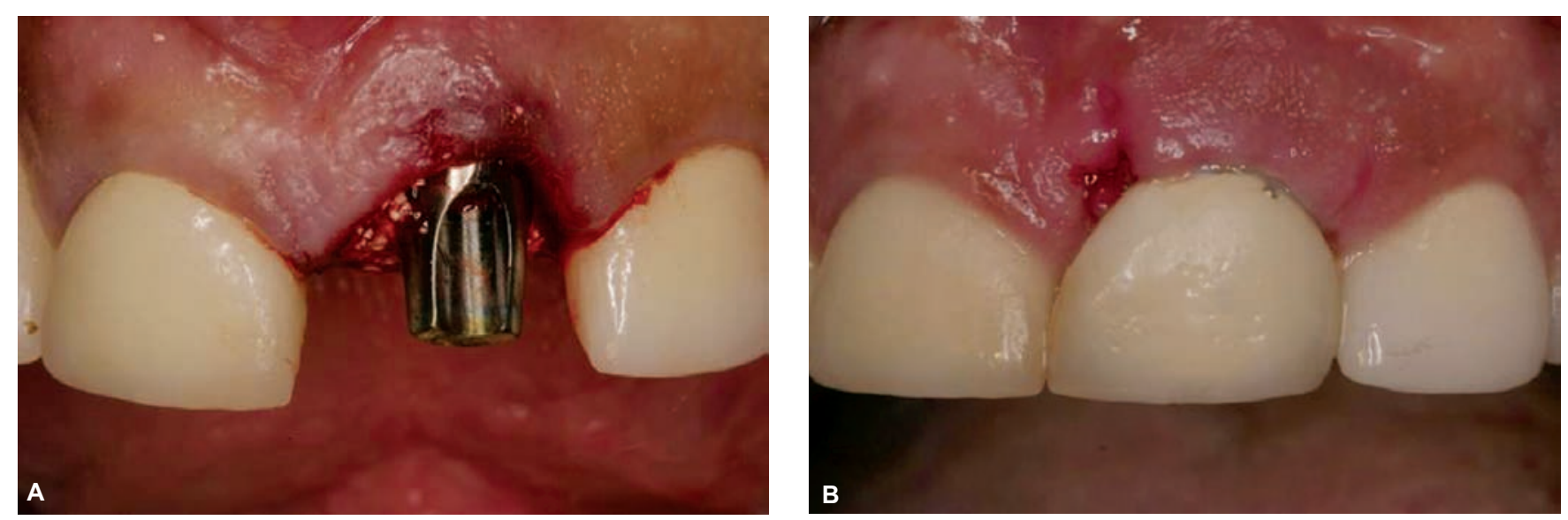

Figs 17A and B: Titanium abutment and temporary

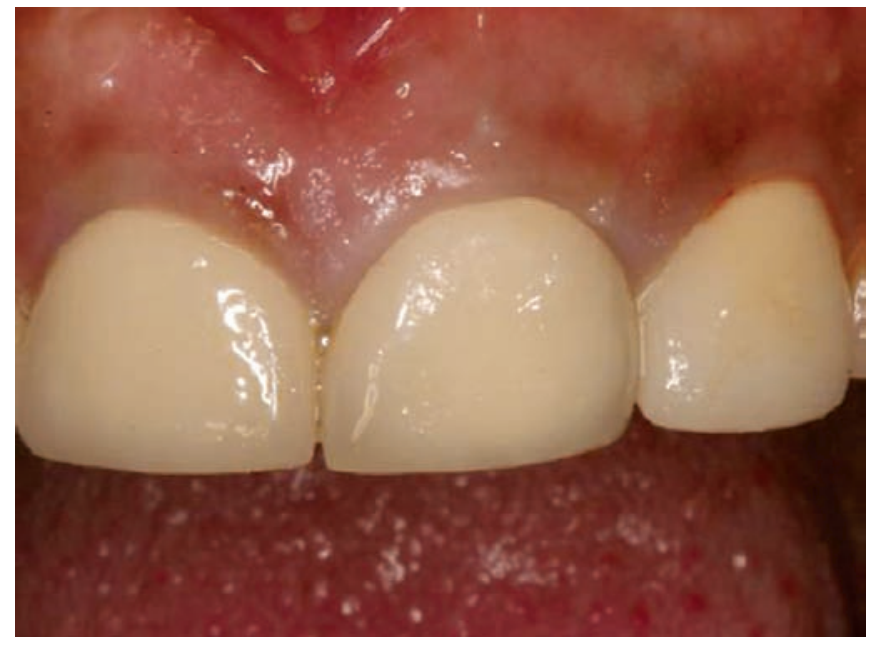

Fig. 18: Final restoration

\section{CASE 5}

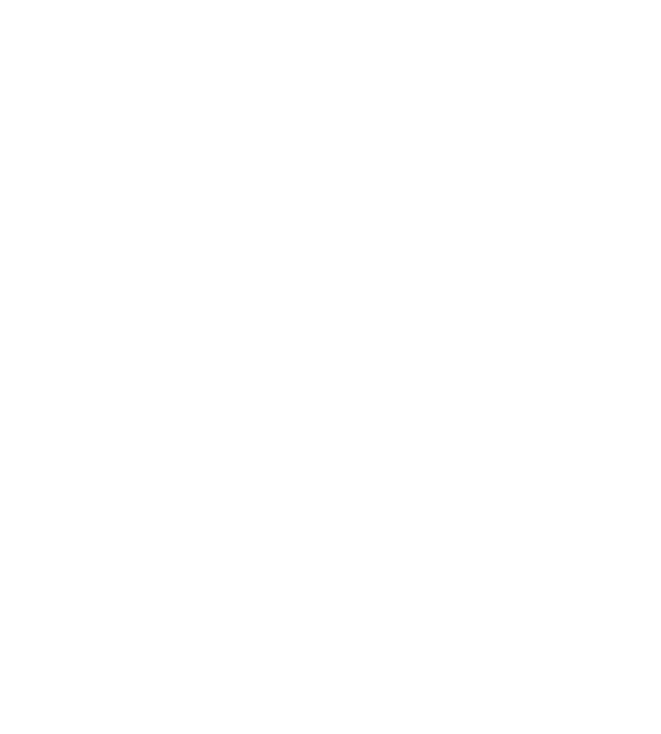

Fig. 19: Preoperative 


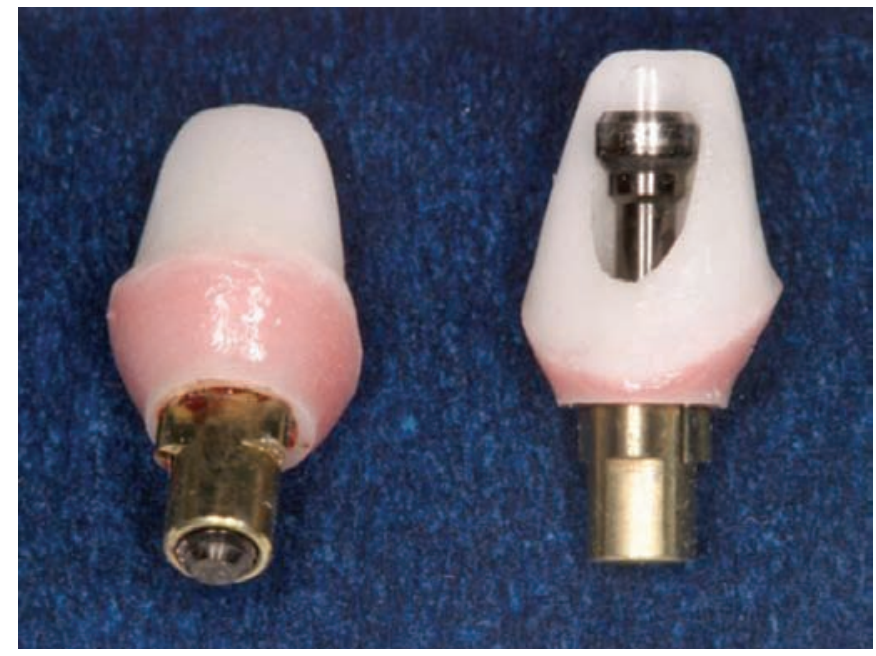

Fig. 20: Ceramic abutments

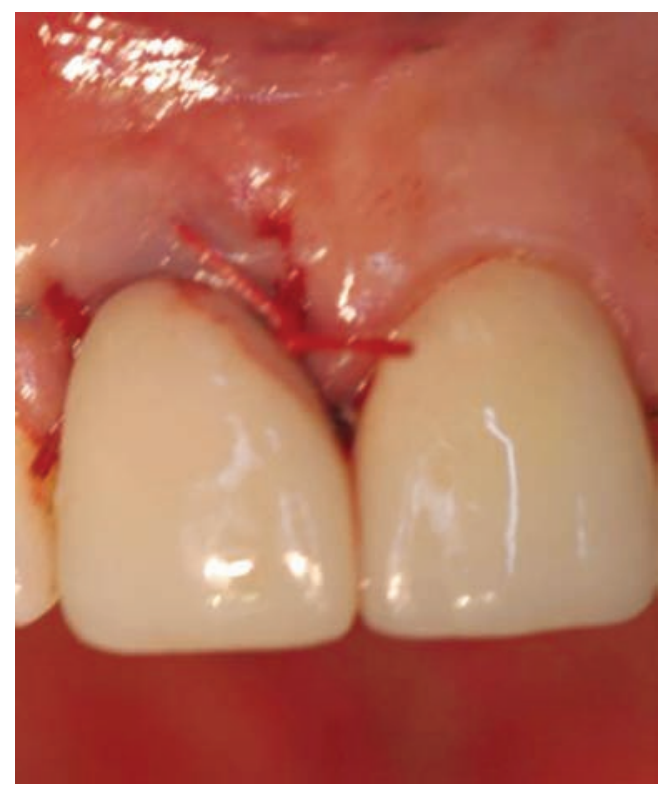

Fig. 22: Provisional restoration

\section{CASE 6}
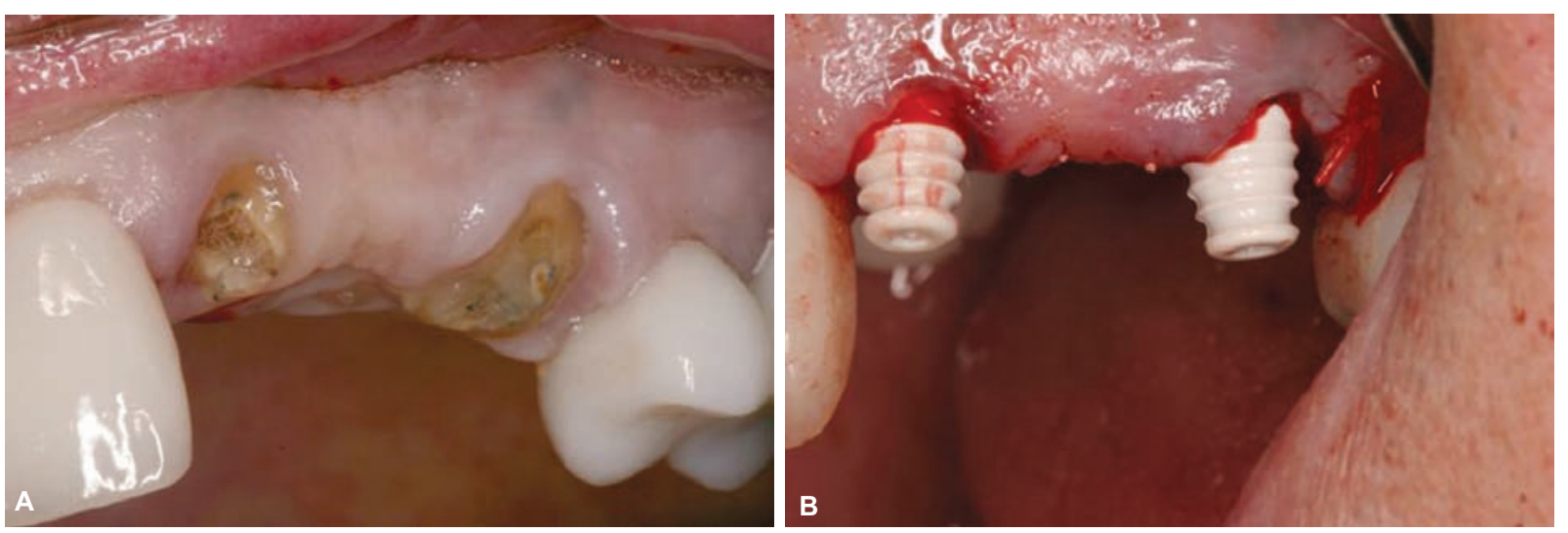

Figs 24A and B: (A) Preoperative (B) Temporary abutments 


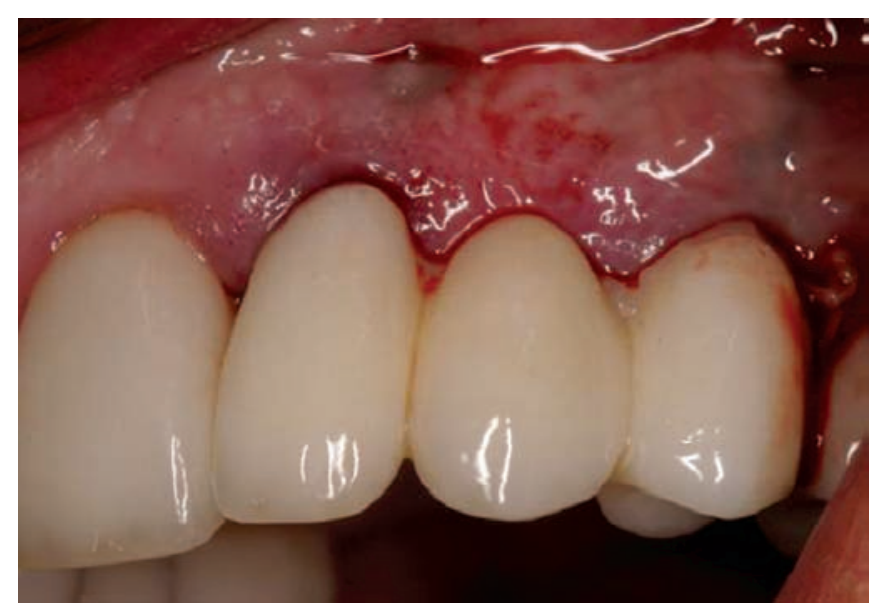

Fig. 25: Patient's bridge adapted

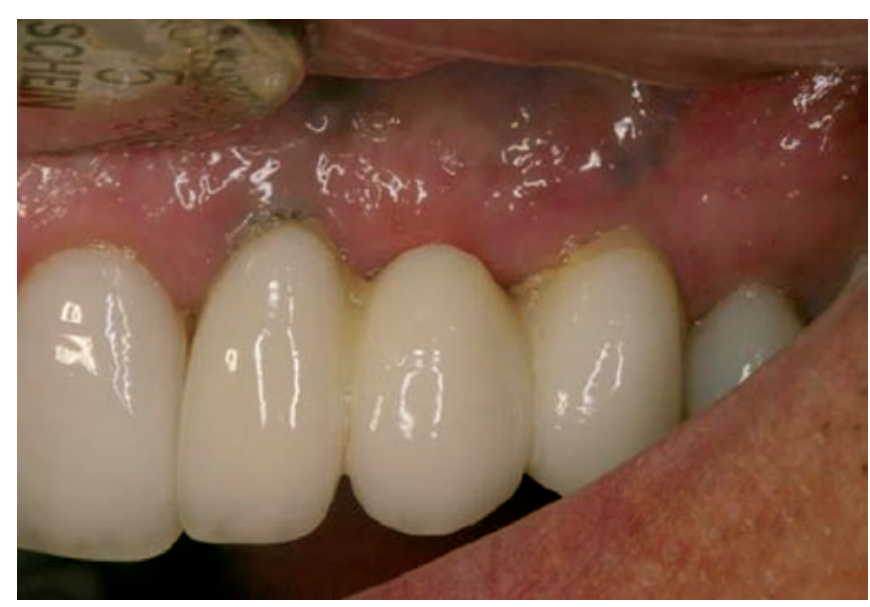

Fig. 26: Seven days postoperative

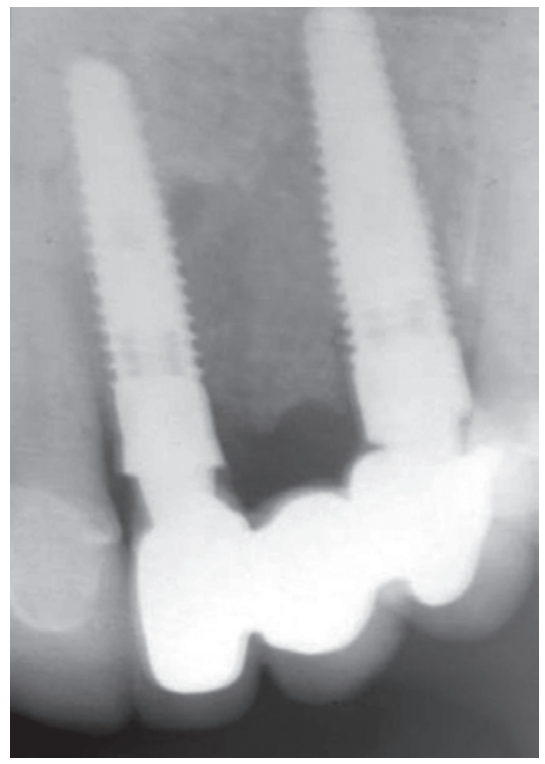

Fig. 27: Five months radiograph

\section{Antibiotic Therapy Initiation}

If the presurgical evaluation reveals any sign of potential acute infection, antibiotic therapy should be initiated 3 to 5 days before surgery.

\section{Preservation of the Bony Receptor Site}

After reflection of the mucoperiosteal tissues, care should be taken to remove the tooth with as little trauma as possible. The teeth may be brittle or even ankylosed to the surrounding bone, particularly when endodontic therapy has been previously carried out. In such cases, a high-speed, contraangle hand piece with a $700 \mathrm{xxl}$ bur (Park dental research, New York, NY) can be used to section the tooth longitudinally. The individual segments can thenbe dissected and carefully removed to preserve the surrounding bony receptor site. The availability of thePeriotome (Nobel Biocare, Yorba Linda, CA) has allowed the removal of residual teeth in an atraumatic manner.

\section{Procedural Delays}

If any purulent exudate is discovered after removing the tooth, placement of the implant should be delayed. In some cases it is not possible to make this determination preoperatively: The surgeon must make the decision to go forward or delay the implant placement interoperatively. Patients should be informed of this possibility preoperatively. In addition, if any purulence is present, no hard or soft tissue grafting procedures should be performed. The affected area should be curetted, irrigated and closed. Appropriate antibiotic therapy should be continued or instituted. The tissue is then allowed to heal for several weeks until soft tissue closure is complete before implant placement is scheduled.

\section{TAPERED IMPLANTS}

Although excellent results can be obtained when placing standard implants in fresh extraction sites, tapered, anatomically shaped implants are the implants of choice for this indication. Instead of having a uniform diameter, such impl ants more closely mimic th e shape of natural tooth roots. They ar e wider at the cervix tha $\mathrm{n}$ the apex. Tapered implants such as the replace system (Nobel Biocare) are available in various lengths 8, 10, 13 and 16 . The implant diameters are $3.5 \mathrm{~mm}, 4.3 \mathrm{~mm}, 5.0 \mathrm{~mm}$ and $6.0 \mathrm{~mm}$. The surface is tiunite. 
When used as replacements for immediate extractions, tapered implants offer a number of advantages. The larger cervical diameter provides better buccal support and helps preserve the root prominence an important benefit in all implant reconstruction and especially so in the esthetic zone of the anterior maxilla. The larger cervical diameter also improves the implant-to-bone interface, which in turn enhances stability and creates a more acceptable emergence profile in relation to the final prosthesis. In many instances, the tapered design may obviate the need to use grafting materials or membranes, thus helping reduce the cost of treatment, since fenestration and dehiscence are greatly reduced over parallel walled implants.

The tapered design also allows the implant to be placed in the same position as the extracted tooth and avoids buccal or labial wall perforation that is common in the anterior maxilla or perforation of the submandiblular and digastric fossa in the mandible when using parallel walled implants. Since the position of the implant is similar to that of the extracted tooth, restoration can be in a more favorable position relative to the opposing arch, thus reducing excessive off-axis loading on the implant. In ad dition, the taper ed implant can be used when a parallel-walled implant is contraindicated in cases with convergent roots. By using a straight or $15^{\circ}$ maximum angle $\mathrm{d}$ abutment for fixed prostheses, the occlusal table will have more acceptable buccolingual dimensions. These dimensions can also be achieved with screw-retained prostheses, if desired.

\section{CONCLUSION}

Whenever the decision has been made to replace a failed tooth with an implant, caeful consideration should be given to placing the implant at the time of th e extraction, rather than delaying placement until after healing of the site has occurred. When the guidelines described in this paper are followed, immediate implant placement can prevent the loss of soft and hard tissues that typically follow extraction. Strong tissue support, in turn, makes the achievement of superior esthetic results more likely. The use of tapered implants and tapered osteotomes to prepare the extraction site increases further the probability of creating an attractive and natural looking emergence profile.

\section{REFERENCES}

1. Babbush CA. Extraction immediate implant placement: 5- year statistical review. Presented at the Annual Meeting of the Academy of Osteointegration, Orlando, 1994.

2. Hahn J. Indication for the use of tapered implants, Den t Surg Products 1997;4:34.

3. Silverstein L, Hahn J. Aesthetic enhancement of anterior dental implants with the use of tapered osteotomes a nd soft-tissue manipulation, Dent Surg Products 1998;6-7:28.

4. Rosenquist B, Grenthe B. Immediate placement of implants into extraction sockets, J Oral Maxillofac Implants 1996;11(2):205.

5. Wong K. Immediate implantation of endosseous dental implants in the posterior maxillae and anatomic advantages for the region: A case report, J Oral Maxillofac Implants 1996;11(4):529.

6. Vizenthum F. Biomechanics for the immediate placement, Acad Osseointegration Newsletter 1996;7(6):97.

7. Block MS, Kent JN. Place ment of endos seouse implants into tooth extraction sites, J Oral Maxillofac Surg 1991;49:1269. 